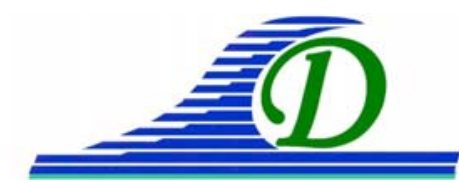

XIII ${ }^{\text {ìmes }}$ Journées Nationales Génie Côtier - Génie Civil

Dunkerque, 2-4 juillet 2014

DOI:10.5150/jngcgc.2014.011 @ Editions Paralia CFL

disponible en ligne - $h$ ttp://www.paralia.fr - available online

\title{
Influence de la profondeur de déferlement des vagues sur la modélisation des transports sédimentaires sur les plages macrotidales
}

\section{Arnaud HEQUETTE ${ }^{1}$, Adrien CARTIER ${ }^{2}$, Thibault OUDART $^{3}$, Philippe LARROUDE ${ }^{3}$}

1. Laboratoire d'Océanologie et de Géosciences, UMR CNRS 8187, Université du Littoral Côte d'Opale, 32 Ave Foch, 62930 Wimereux, France.

arnaud.hequette@univ-littoral.fr

2. Géodunes, 10 rue Bel Air, 59240 Dunkerque, France.

3. Laboratoire des Ecoulements Géophysiques et Industriels, UMR CNRS 5519, Université de Grenoble, B.P. 53, 38041 Grenoble Cedex, France.

\section{Résumé :}

Des mesures hydrodynamiques et de flux sédimentaires longitudinaux ont été réalisées sur une plage macrotidale à barres intertidales. Les résultats ont montré que le déferlement des vagues se produit à des profondeurs moindres que ce qui est généralement avancé dans la littérature, l'indice de déferlement $\left(H_{s b} / h_{b}\right)$ étant généralement compris entre 0,2 et 0,4. La comparaison des flux mesurés in situ avec des flux sédimentaires calculés à l'aide d'un modèle de transport de sédiments utilisant l'équation de BIJKER (1968) a révélé que l'indice de déferlement choisi dans la modélisation a une influence majeure sur la validité des résultats, les meilleurs résultats ayant été obtenus en utilisant des indices $H_{s b} / h_{b}$ correspondants aux valeurs observées à partir desquelles les vagues commencent à déferler.

Mots-clés : Hydrodynamique littorale, Indice de déferlement, Transport sédimentaire littoral, Modélisation des flux sédimentaires, Plages macrotidales.

\section{Introduction}

Comparativement aux plages où la marée ne joue qu'un rôle faible ou modéré, les plages macrotidales n'ont fait l'objet que de quelques études pour tenter d'y mesurer les transports sableux (e.g., LEVOY et al., 1994 ; SEDRATI \& ANTHONY, 2007 ; CARTIER et al., 2013). Sur les plages à fort marnage, la nature et l'intensité des processus hydro-sédimentaires sont caractérisés par une grande variabilité en raison de la présence de barres sableuses et de dépressions (bâches) dans la zone intertidale, mais aussi à cause des fortes fluctuations du niveau de marée qui induisent une translation horizontale des différentes zones hydrodynamiques littorales (zone de levée des vagues (shoaling), de déferlement et de surf) (LEVOY et al., 2001). La complexité des transports sédimentaires est par conséquent encore plus grande sur les plages macrotidales du fait de cette perpétuelle migration de la zone de déferlement et de la zone de surf qui est responsable de variations continuelles des conditions de 


\section{Thème 1 - Hydrodynamique côtière}

déferlement de la houle à travers les barres et les bâches de la zone intertidale (CARTIER \& HEQUETTE, 2013).

Il est largement admis que les quantités de sédiments transportés par la dérive littorale dépendent fortement de la hauteur de la houle au déferlement, mais la mesure de cette hauteur de déferlement est un exercice difficile, notamment parce que la position du point de déferlement varie en fonction des conditions de houle, mais aussi des fluctuations du niveau d'eau dans les mers à marées. Un indice théorique de déferlement est par conséquent couramment utilisé dans les modèles numériques de transport sédimentaire littoral afin d'estimer la hauteur de la houle au déferlement. Un des indices de déferlement les plus utilisés est l'indice de McCOWAN (1894) selon lequel le rapport de hauteur de vague sur la profondeur au déferlement $\left(H_{s b} / h_{b}\right)$ est égale à 0,78 . De nombreuses études ont cependant montré que les vagues pouvaient déferler à des profondeurs très différentes de celles correspondant à l'indice théorique de McCowan, le rapport $H_{s b} / h_{b}$ dépendant notamment de paramètres comme la pente de la plage et la cambrure de la houle (SALLENGER \& HOLLMAN, 1985 ; CAMENEN \& LARSON, 2007). Des indices de déferlement $H_{s b} / h_{b}$ compris entre 0,3 et 0,5 sont fréquemment proposés pour les plages macrotidales, (MASSELINK et al., 2006 ; SEDRATI \& ANTHONY, 2007), mais ces valeurs ne sont basées que sur un nombre très limités de mesures in situ en conditions de fort marnage.

Cet article présente les résultats d’une étude menée sur une plage macrotidale du Nord de la France dans le but d'analyser la variabilité du déferlement à travers la zone intertidale et d'évaluer l'influence de la hauteur des vagues et de la profondeur au déferlement sur les transports sédimentaires longitudinaux engendrés par la dérive littorale. La comparaison de mesures in situ des flux sédimentaires avec des flux calculés à l'aide d'un modèle numérique de transport a permis de déterminer l'indice de déferlement le plus approprié pour la modélisation des transports sédimentaires longitudinaux sur la plage étudiée.

\section{Site d'étude}

Cette étude a été réalisée sur une plage sableuse $\left(D_{50}=0,17 \mathrm{~mm}\right)$ située à Zuydcoote sur le littoral du Nord de la France (figure 1A). Le site de mesures est caractérisé par un estran de faible pente $(\tan \beta=0,014)$, d'environ $450 \mathrm{~m}$ de largeur, marqué par la présence de plusieurs barres intertidales de hauteur et largeur variables (figure 1B). La plage de Zuydcoote fait face à la mer du Nord et est exposée à des houles d'énergie faible à modérée suite à leur réfraction sur les bancs sableux pré-littoraux et les faibles pentes qui caractérisent l'avant-côte (HEQUETTE et al., 2009).

La marée est de type semi-diurne et le marnage est macrotidal, l'amplitude moyenne de la marée à Dunkerque étant d'environ 5,5 m en vive eau et de 3,5 m en morte eau. Ce fort marnage est responsable de forts courants de marée qui s’écoulent parallèlement au 


\section{XIII ${ }^{\text {èmes }}$ Journées Nationales Génie Côtier - Génie Civil \\ Dunkerque, 2-4 juillet 2014}

rivage dans la zone côtière, mais dont l'intensité décroit fortement du bas vers le haut de plage (CARTIER \& HEQUETTE, 2013).

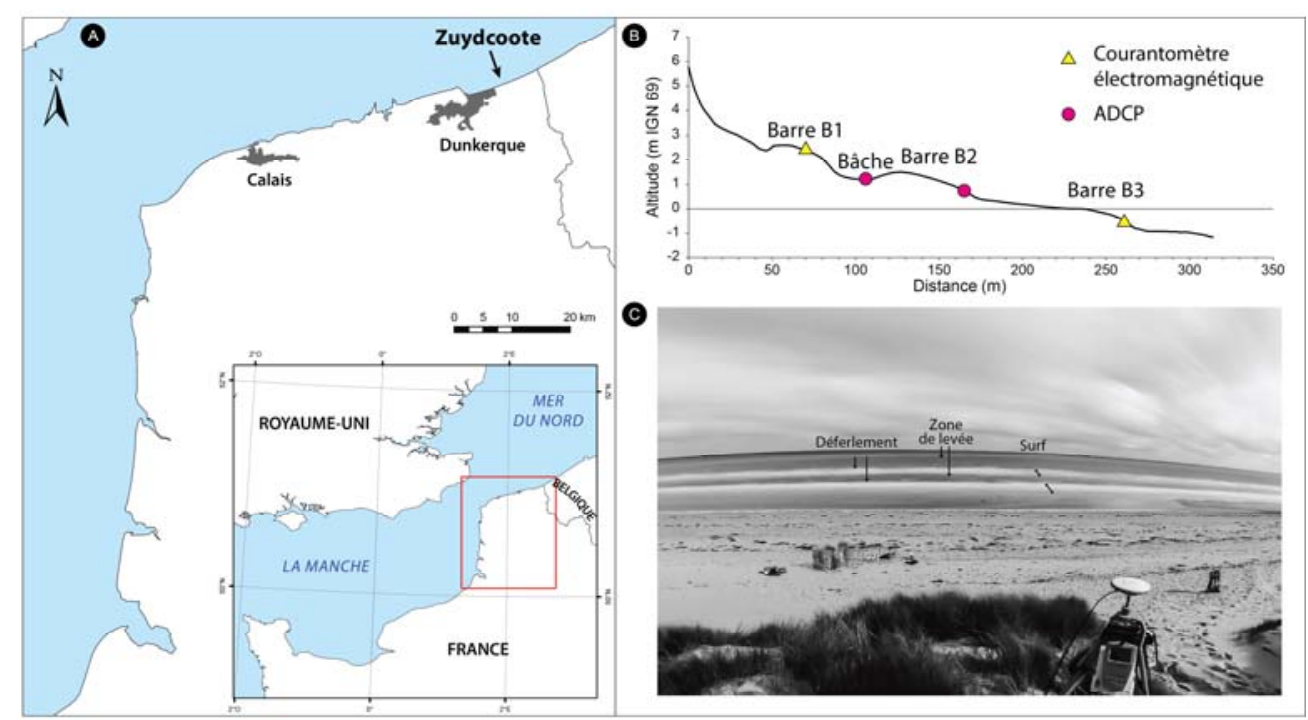

Figure 1. A) Localisation du site d'étude ; B) Positionnement des appareils de mesures dans la zone intertidale ; C) Exemple d'enregistrement vidéo de la zone côtière.

\section{Méthodologie}

La méthode utilisée repose sur l'acquisition simultanée de données hydrodynamiques et de mesures in situ de flux sédimentaires à l'aide de pièges à sédiments. Les mesures hydrodynamiques ont été réalisées en juin 2013 à l'aide de deux courantomètreshoulographes électromagnétiques et de deux profileurs de courant (ADCP) disposés sur trois barres intertidales et dans une bâche le long d'une radiale perpendiculaire au trait de côte (figure 1B). Ces appareils permettent d'obtenir des mesures des paramètres de houle (hauteur significative $\left(H_{s}\right)$, période et direction) et de courant (vitesse et direction du courant moyen, vitesse de la composante transversale et longitudinale du courant), ainsi que la hauteur moyenne de la colonne d'eau. Les courants ont été mesurés à proximité du fond: $0,15 \mathrm{~m}$ au-dessus du fond dans le cas des courantomètres électromagnétiques et $0,2 \mathrm{~m}$ dans le pour les ADCP (hauteur de la première cellule audessus de l'appareil). La fréquence d'acquisition des données hydrodynamiques a été de $2 \mathrm{~Hz}$ pour tous les appareils qui ont été programmés pour enregistrer des données toutes les 15 minutes pendant 8,5 minutes (courantomètres électromagnétiques) et pendant 10 minutes (ADCP).

Les mesures des flux sédimentaires ont été réalisées en utilisant des pièges à sédiments qui permettent de mesurer le transport sédimentaire en suspension a plusieurs niveaux dans la colonne d'eau et proche du fond grâce à cinq filets d'une maille de $63 \mu \mathrm{m}$ repartis régulièrement sur une hauteur de 1,43 m. Les pièges à sédiments sont placés 


\section{Thème 1 - Hydrodynamique côtière}

face au courant longitudinal pendant une durée de 10 minutes. Les flux de sédiments ont été déterminés au niveau de chaque piège, puis intégré sur la colonne d'eau en suivant la procédure préconisée par ROSATI et KRAUS (1989). Toutes les mesures de flux ont été effectuées au voisinage direct des appareils courantométriques. Les mesures in situ de flux sédimentaires ont été comparées aux résultats de modélisation des flux sédimentaires basés sur le couplage de trois codes (Tomawac pour la propagation de la houle, Telemac 2D pour les courants et Sisyphe pour l'évolution du fond) et en utilisant l'équation de transport de BIJKER (1968).

Une caméra vidéo programmée pour prendre un cliché toutes les 10 secondes a été installée en haut de dune à l'arrière de la plage à une hauteur de $10 \mathrm{~m}$ au-dessus du niveau de marée moyen. Des images correspondant à une moyenne de 60 clichés consécutifs de la plage et de la zone côtière ont permis de localiser la zone de surf, de déferlement et de levée lors de chaque période de mesures hydrodynamiques réalisée en plein jour (figure 1C), ce qui a permis de savoir quel type de processus hydrodynamique (levée des vagues, déferlement, surf) agissait au niveau de chaque appareil courantométrique lors de chaque période d'enregistrement.

\section{Résultats}

\subsection{Variations des processus hydrodynamiques dans la zone intertidale}

La campagne de mesures a été caractérisée par des conditions de houle d'énergie modérée, la hauteur des vagues ayant varié de moins de $0,20 \mathrm{~m}$ à $0,93 \mathrm{~m}$, cette hauteur maximale ayant été atteinte sur la barre B3 en bas de plage. Les hauteurs de vagues montrent une forte variabilité à travers l'estran, les hauteurs diminuant vers le haut de plage en raison de la dissipation de l'énergie des vagues au fur et à mesure de leur propagation au-dessus des faibles profondeurs de l'estran ainsi qu'en raison de leur déferlement sur les barres intertidales.

C’est au niveau de la barre B3, située la plus au large, qu'ont été observées les plus importantes variations de conditions hydrodynamiques, la barre étant successivement soumise aux processus de levée de vagues, de déferlement et de surf pendant chaque cycle de marée (figure 2). A l'inverse, la barre B1 située en haut de plage est essentiellement affectée par des processus de surf et de déferlement, sans que les processus de levée des vagues n'agissent sur cette partie haute de la plage. On observe presque exclusivement des conditions de levée des vagues dans les bâches, car les vagues se reforment dans la dépression suite au déferlement sur la barre inférieure, ce qui explique les faibles valeurs de $H_{s} / h$ (généralement inférieure à 0,3 ) qui résultent à la fois de la diminution de hauteur de vague après le déferlement et de l'augmentation de la profondeur dans la bâche (figure 3A). Les rapports $H_{s} / \mathrm{h}$ sont généralement plus élevés sur les barres, car l'épaisseur de la tranche d'eau y est plus faible que dans les bâches, mais aussi parce que la hauteur des vagues augmente avec le déferlement qui se 


\section{XIII ${ }^{\text {èmes }}$ Journées Nationales Génie Côtier - Génie Civil \\ Dunkerque, 2-4 juillet 2014}

produit préférentiellement sur les barres. Ceci est particulièrement le cas au niveau de la barre B2, et surtout de la barre B1, qui ont été exclusivement soumises à des processus de déferlement et de surf pendant ces mesures (figure 3A). Sur la barre B3, les mesures de $H_{s} / \mathrm{h}$ ont révélé des valeurs similaires à celles mesurées sur la barre B2, mais aussi des valeurs nettement plus faibles $\left(H_{s} / h<0,25\right)$ qui ont été enregistrées lorsque la barre était en zone de levée des vagues à la faveur de l'augmentation de la tranche d'eau due à la marée.

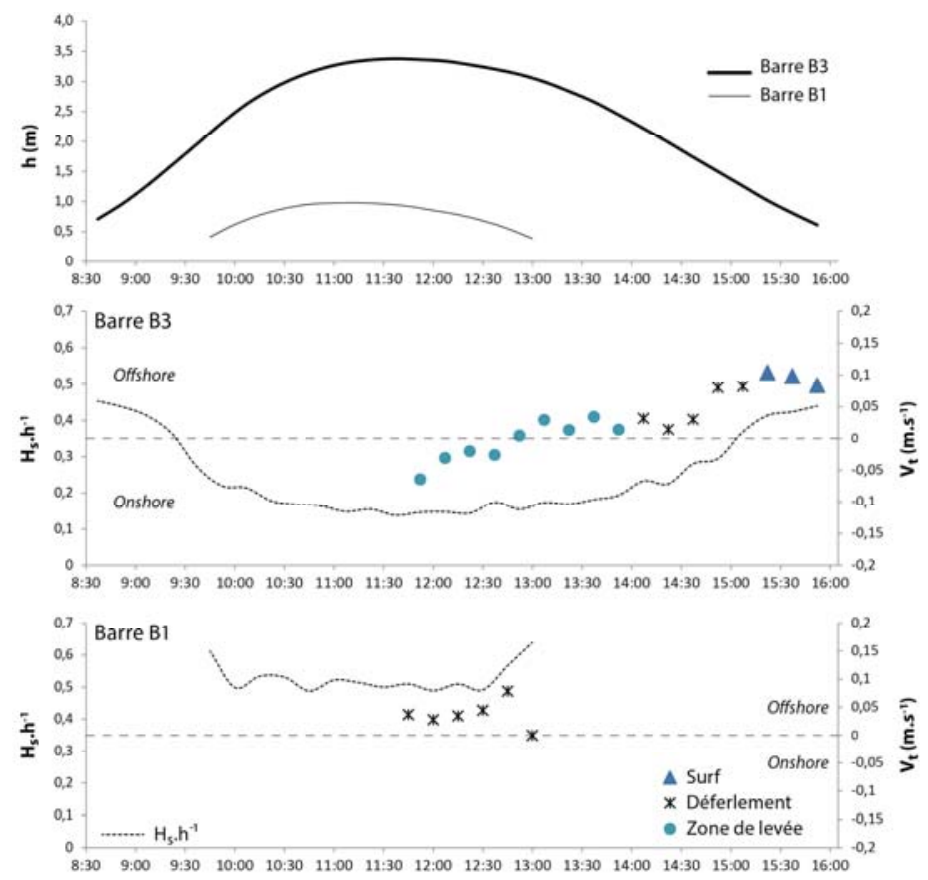

Figure 2. Exemple de variations de la profondeur moyenne $(h)$, du rapport $\left(H_{s} / h\right)$ et de la vitesse moyenne du courant transversal $\left(V_{t}\right)$ mesurées le 05/06/2013 au niveau de la barre B1 et B3 (voir figure 1B pour la localisation des appareils).

L'analyse des vitesses moyennes du courant transversal $\left(V_{t}\right)$ a également montré qu'il existait une grande variabilité dans les directions de courants mesurés à différents niveaux de l'estran (figure 3A). Dans la bâche, le courant transversal n'est dirigé que vers la côte (onshore) avec une vitesse moyenne pouvant dépasser $0,2 \mathrm{~m} \mathrm{~s}^{-1}$, alors que sur les barres le courant transversal peut être dirigé vers la côte ou vers le large (offshore), tout dépendant de la position de la barre sur l'estran, de la hauteur de la houle et du niveau d'eau au moment de la mesure. Au niveau de la barre B3, le courant transversal est dirigé soit vers la côte soit vers le large lors de conditions de levée des vagues, mais sa vitesse est faible $\left(<0,07 \mathrm{~m} \mathrm{~s}^{-1}\right)$ ou nulle (figure 3B). Des vitesses plus élevées ont été enregistrées sur cette barre et sur les deux autres barres lorsqu'elles étaient soumises à des processus de déferlement ou de surf, les plus fortes vitesses enregistrées correspondant principalement à des courants dirigés vers le large (figure 
3A). Ces courants dont la vitesse a pu dépasser $0,2 \mathrm{~m} \mathrm{~s}^{-1}$ au niveau de la barre B2 correspondent vraisemblablement à des courants de retour qui se développent préférentiellement en zone de surf et de déferlement (GREENWOOD \& OSBORNE, 1991 ; MASSELINK et al., 2006). Ces courants vers le large sont moins forts sur la barre B1, ce qui s'explique par le fait que les vagues (qui sont à l'origine de ces courants de retour) ont dissipé une grande partie de leur énergie à travers l'estran lorsqu'elles atteignent cette barre de haut de plage (LEVOY et al., 2001 ; CARTIER \& HEQUETTE, 2013).
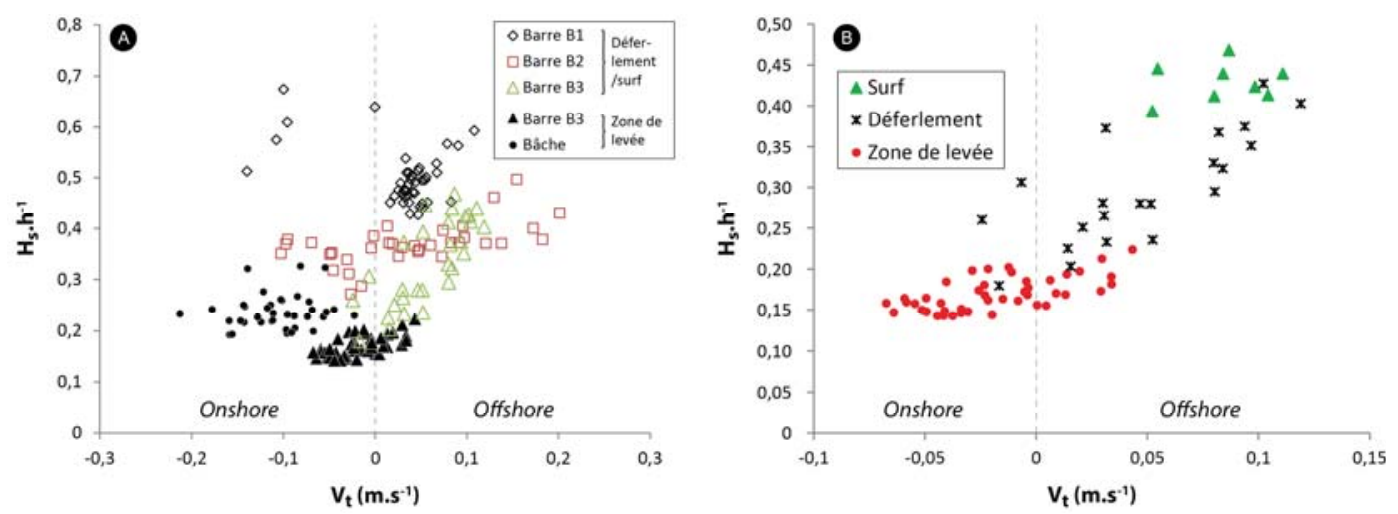

Figure 3. Relation entre le rapport $\left(H_{s} / h\right)$ et la vitesse moyenne du courant transversal $\left(V_{t}\right)$ pour les mesures réalisées pendant les enregistrements vidéo ; A) Ensemble des mesures réalisées sur les barres et dans la bâche; B) mesures réalisées sur la barre B3.

Les résultats obtenus pendant cette campagne de mesures montrent que les barres intertidales sont affectées par des processus de déferlement et de surf lorsque le rapport $H_{s} / \mathrm{h}$ est compris entre approximativement 0,2 et 0,7 , les valeurs de $H_{s} / \mathrm{h}$ étant plutôt compris entre 0,3 et 0,5 pour la barre B2 et entre environ 0,45 et 0,7 pour la barre B1 (figure 3A). Ces deux barres n'ayant été localisées qu'en zone de déferlement ou de surf pendant ces mesures, il n'est pas possible de déterminer une valeur de $H_{s} / \mathrm{h}$ qui correspondrait à une valeur limite à partir de laquelle les vagues commenceraient à déferler sur cette partie de la plage. Des seuils entre les différentes zones hydrodynamiques peuvent cependant être établis à partir des enregistrements réalisés sur la barre B3 qui a été soumise aux processus de déferlement et de surf, mais aussi de levée des vagues (figure 3B). Au niveau de cette barre, le déferlement n'est généralement pas observé tant que le rapport $H_{\mathcal{S}} / \mathrm{h}$ n'atteint pas une valeur minimale d'environ 0,2 , le déferlement continuant de se produire jusqu'à des valeurs de 0,4 , les processus de surf se produisant plutôt lorsque $H_{\mathcal{s}} / \mathrm{h}$ est compris entre 0,4 et 0,5.

\subsection{Modélisation des flux sédimentaires longitudinaux}

Les résultats de la modélisation des transports sédimentaires obtenus en utilisant différents indices de déferlement $\left(H_{s b} / h_{b}\right)$ montrent que les flux calculés varient de façon 


\section{XIII ${ }^{\text {èmes }}$ Journées Nationales Génie Côtier - Génie Civil \\ Dunkerque, 2-4 juillet 2014}

extrêmement importante en fonction de l'indice $H_{s b} / h_{b}$ choisi (figure 4). L'utilisation d'un indice de déferlement $>0,2$ induit une très forte surévaluation des flux longitudinaux, les flux calculés étant plusieurs dizaines de fois supérieurs aux flux mesurés avec un indice de 0,3 et surtout de 0,78 (indice de McCowan). Les meilleurs résultats ont été obtenus avec des $H_{s b} / h_{b}$ de 0,15 et de 0,2 , les flux calculés étant alors nettement plus proches des flux mesurés in situ (figure 4). Même si la calibration du modèle numérique utilisé pour calculer les transports sédimentaires nécessite d'être améliorée, il est notable de constater que les meilleurs résultats ont été obtenus en utilisant des valeurs de $H_{s b} / h_{b}$ qui sont proches du seuil observé sur le terrain entre des conditions de levée des vagues et de déferlement.
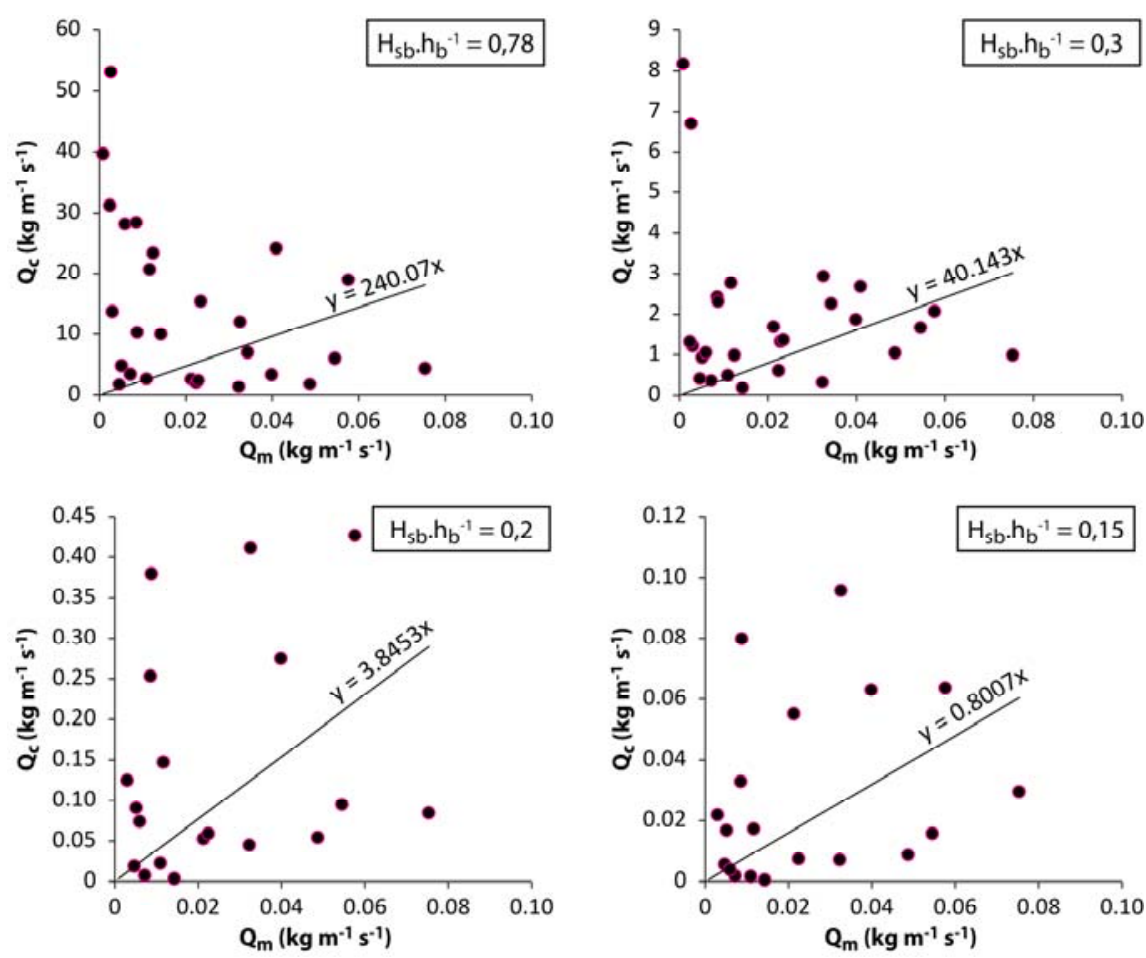

Figure 4. Comparaison entre les flux sédimentaires longitudinaux mesurés $\left(Q_{m}\right)$ et les flux sédimentaires calculés $\left(Q_{c}\right)$ pour différents indices de déferlement $\left(H_{s b} / h_{b}\right)$.

\section{Conclusions}

Les résultats obtenus dans cette étude montrent que le déferlement sur une plage à barres intertidales de faible pente se produit à des profondeurs moindres que celles prévues par un indice théorique comme celui de McCowan, les indices de déferlement $\left(H_{s b} / h_{b}\right)$ étant généralement compris entre 0,2 et 0,4 . La comparaison des flux mesurés in situ avec les flux calculés a mis en évidence l'importance du choix de l'indice de déferlement dans la modélisation des transports sur ce type de plage, les meilleurs résultats ayant été obtenus avec des indices $H_{s b} / h_{b}$ de 0,15 et 0,2 qui correspondent aux valeurs à partir desquelles les vagues commencent à déferler sur la plage étudiée. 


\section{Références bibliographiques}

BIJKER E. (1968). Littoral drift as function of waves and current. $11^{\text {th }}$ International Conference on Coastal Engineering, Londres, pp 415-435.

CAMENEN B, LARSON M (2007). Predictive formulas for breaking depth index and breaker type. Journal of Coastal Research, Vol. 23, pp 1028-1041.

CARTIER A., HEQUETTE A. (2013). The influence of intertidal bar-trough morphology on sediment transport on macrotidal beaches, Northern France. Zeitschrift für Geomorphologie, Vol. 57, pp 325-347.

CARTIER A., LARROUDE P., HEQUETTE A. (2013). Longshore sediment transport measurements on sandy macrotidal beaches compared with sediment transport formulae. In: A. Manning (ed.), Sediment Transport, InTech Publishing, pp 37-58. http://dx.doi.org/10.5772/51023

GREENWOOD B, OSBORNE P.D. (1991). Equilibrium slopes and cross-shore velocity asymmetries in a storm-dominated, barred nearshore system. Marine Geology, Vol. 96, pp 211-235. http://dx.doi.org/10.1016/0025-3227(91)90148-W

HEQUETTE A., RUZ M.H., MASPATAUD A., SIPKA V. (2009). Effects of nearshore sand bank and associated channel on beach hydrodynamics: implications for beach and shoreline evolution. Journal of Coastal Research, Sp. Issue 56, pp 59-63.

LEVOY F., MONTFORT O., ROUSSET H. (1994). Quantification of longshore transport in the surf zone on macrotidal beaches. Fields experiments along the western coast of Cotentin (Normandy, France). 24th International Conference on Coastal Engineering, Kobe, Japon, pp 2282-2296.

LEVOY F., MONFORT O., LARSONNEUR C. (2001). Hydrodynamic variability on megatidal beaches, Normandy, France. Continental Shelf Research, Vol. 21, pp 563586. http://dx.doi.org/10.1016/S0278-4343(00)00128-X

McCOWAN J (1894). On the highest wave of permanent type. Philosophical Magazine, Vol. 38 (Ser. 5), pp 351-358.

MASSELINK G., KROON A., DAVIDSON-ARNOTT R. (2006). Morphodynamics of intertidal bars in wave-dominated coastal settings - A review. Geomorphology, Vol. 73, pp 33-49. http://dx.doi.org/10.1016/j.geomorph.2005.06.007

ROSATI J.D., KRAUS N.C. (1989). Development of a portable sand trap for use in the nearshore. Department of the Army, U.S. Corps of Engineers. Technical report CERC, pp 89-91, 181 p.

SALLENGER A.H., HOLLMAN R.A. (1985). Wave energy saturation on a natural beach of variable slope. Journal of Geophysical Research, Vol. 90 (C6), pp 1193911944. http://dx.doi.org/10.1029/JC090iC06p11939

SEDRATI M., ANTHONY E.J. (2007). Storm-generated morphological change and longshore sand transport in the intertidal zone of a multi-barred macrotidal beach. Marine Geology, Vol. 244, pp 209-229. http://dx.doi.org/10.1016/j.margeo.2007.07.002 